Brit. Heart f. 1966, 28, 382.

\title{
Hypertension due to Subadventitial Fibrosis of the Renal Artery
}

\author{
J. A. LYTTLE^ AND W. T. E. McCAUGHEY† \\ From the Royal Victoria Hospital, Belfast, and the Department of Pathology, The Queen's University of Belfast
}

Increasing awareness of the importance of renal artery stenosis as a cause of hypertension has focused attention on the lesions causing stenosis. Atheromatous plaques located at the origin or in the proximal third of the renal artery have been the most common finding (Brown et al., 1960; Morris and De Bakey, 1962). Less frequently reported lesions have included muscular or fibromuscular hyperplasia, mural fibrosis, intimal fibrosis, primary thrombosis, emboli, dissecting aneurysms, and compression of the artery by fibrous bands or tumour (McCormack, 1961; Hunt et al., 1962; D'Abreu and Strickland, 1962).

Fibromuscular hyperplasia is of particular interest because of its frequency as a cause of hypertension in young adults. The hyperplasia usually involves the distal two-thirds of the artery. The most striking feature is the presence of alternating zones of fibromuscular thickening and deficiency of the media giving the characteristic "string of beads" appearance with renal arteriography (Hunt et al., 1962; Wylie, Perloff, and Wellington, 1962). A few reports mention another type of arterial lesion that appears to be equally distinctive. It is characterized by a prominent zone of fibrosis between the adventitia and muscle coats (Wylie et al., 1962; Wood and Borges, 1963; Wellington, 1963; Sutton et al., 1963). Two of the cases described by Wellington also showed fibromuscular hyperplasia, and he suggested that the conditions might be related.

In the past 3 years, 130 young hypertensive patients were investigated in this hospital to exclude renal artery stenosis as a cause of their hypertension. In 15 patients unilateral renal artery stenosis was confirmed at operation. In a further 12 patients

Received June 24, 1965.

$\star$ Present address: The National Hospital, Queen Square, W.C.1.

$t$ Present address: School of Pathology, Trinity College, Dublin. who did not come to operation this diagnosis was considered probable. Fibrous or fibromuscular thickening of the renal arteries was found in 10 of the operated group and in 2 of these the stenosis was due to subadventitial fibrosis. In view of the apparent rarity of this condition, we report here the clinical, radiological, and pathological findings in these 2 cases.

\section{CASE Reports}

Case 1. A 28-year-old woman shorthand typist was first seen in 1957, at the age of 23, complaining of morning headache, nervousness, and intermittent swelling of her ankles during the previous two years. There was no history of previous renal disease and no family history of hypertension. The blood pressure was $195 / 130 \mathrm{~mm}$. Hg. The aortic component of the 2nd heart sound was accentuated. The fundi showed a grade 1 angiospastic retinopathy, the kidneys were not palpable, and the femoral pulses were normal. Chest radiograph showed a normal cardiac outline and an electrocardiogram was normal. Blood urea was $34 \mathrm{mg} . / 100 \mathrm{ml}$. Urinalysis was normal. The ESR (Westergren) was $16 \mathrm{~mm}$. in the first hour. A phentolamine test was negative. An intravenous pyelogram was reported to be normal.

A bilateral lumbo-dorsal sympathectomy was carried out, but after one week the blood pressure had returned to pre-operative levels. Between 1958 and 1962 the blood pressure was controlled with reserpine, hydrallazine, and mecamylamine.

In 1962 a right subcostal bruit was noted and the patient admitted for further investigation. The blood urea was $28 \mathrm{mg}$. $/ 100 \mathrm{ml}$. Serum potassium $3.8 \mathrm{mEq} /$ litre and $\mathrm{CO}_{2} \mathrm{CP} 26 \mathrm{mEq} /$ litre. A repeat intravenous pyelogram showed that the right kidney was $1 \mathrm{~cm}$. smaller than the left in bipolar diameter, the calyceal pattern was normal, and there was a slightly greater concentration of contrast in the right renal pelvis at 5 minutes. A radio-hippuran renogram showed peak counts over the right renal area less than those over the left renal area, indicating significant asymmetry of renal function. Differential renal function studies during 


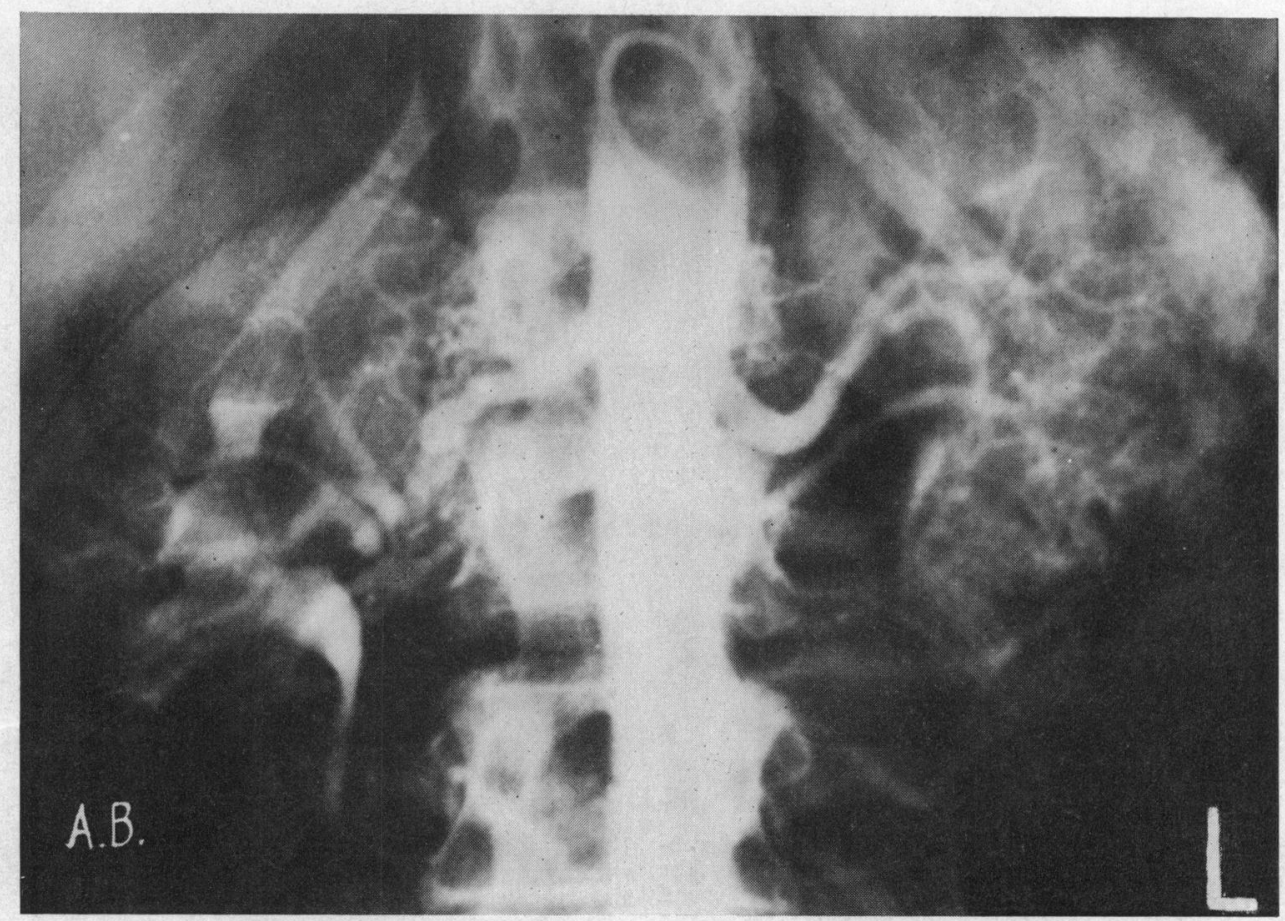

FIG. 1.-Renal arteriogram (Case 1), showing segmental narrowing ("string of beads" appearance) of the distal portion of the main right renal artery with a collateral circulation at the hilum of the kidney.

water diuresis showed an 80 per cent greater tubular reabsorption of sodium by the right kidney than by the left, suggesting right renal artery stenosis. A renal arteriogram (Fig. 1) showed segmental narrowing of the middle and distal portion of the right renal artery with a collateral circulation of capsular vessels around the hilum. At operation on January 10, 1963, the main right renal artery was normal for the first $4 \mathrm{~cm}$. Beyond this there was considerable thickening of the wall followed by a dilated segment. A further area of thickening was present at the bifurcation of the main renal artery. Pressure in the aorta was $160 / 115 \mathrm{~mm} . \mathrm{Hg}$ and in the post-stenotic dilatation $40 / 32 \mathrm{~mm}$. $\mathrm{Hg}$, when measured simultaneously. An apparently normal aberrant renal artery was identified entering the hilum. Due to the extent of the lesion, no reconstructive surgery was considered possible and a right nephrectomy was carried out, the right renal artery being sectioned approximately $2 \mathrm{~cm}$. from its distal end. The blood pressure was $130 / 85 \mathrm{~mm}$. $\mathrm{Hg}$, 24 hours after operation and $110 / 90 \mathrm{~mm}$. $\mathrm{Hg} 2$ years later.

Pathology. The kidney weighed $130 \mathrm{~g}$. and showed no scarring or other lesion. At the point of section of the main renal artery there was a short segment $(0.5$ $\mathrm{cm}$.) where there was marked narrowing of the lumen due to eccentric thickening of the wall. Distal to this the lumen was slightly dilated and the wall was thin for a distance of $1 \mathrm{~cm}$. Just proximal to the bifurcation the lumen became slightly narrowed. The main branches of the artery and the aberrant artery noted at operation appeared normal.

Histology. The renal parenchyma appeared unremarkable apart from slight prominence of the juxtaglomerular apparatus at a few points and slight sclerosis of the smaller intrarenal arteries. In the stenosed segment of main renal artery the wall was grossly thickened by eccentric subadventitial fibrosis (Fig. 2). Elastic tissue staining showed the external elastica on the outer aspect of the fibrosis, but in some areas there were external elastica fibres in the substance of or on the inner surface of the subadventitial fibrosis. In many places, however, the fibrosis replaced the outer media, as was shown by the inclusion of occasional bundles of medial muscle in its substance (Fig. 3). The inner media showed patchy fibrosis but was preserved as a continuous layer. At several points large sinusoidal vascular spaces with an elastic lamina were seen in the media. The internal elastica was thickened in places and there were small cushions of intimal fibrosis. The adventitia appeared unremarkable. The subadventitial fibrosis persisted distal to the stenosed segment, and here there was considerable variation in the thickness of artery wall, the appearances being similar to those of fibromuscular hyperplasia. Thickened segments bearing hyperplastic bundles of medial muscle alternated with areas of marked thinning, where sometimes the vessel wall was largely composed of fibrous tissue (Fig. 4). At several points where fibrosis was slight, it was seen to be 


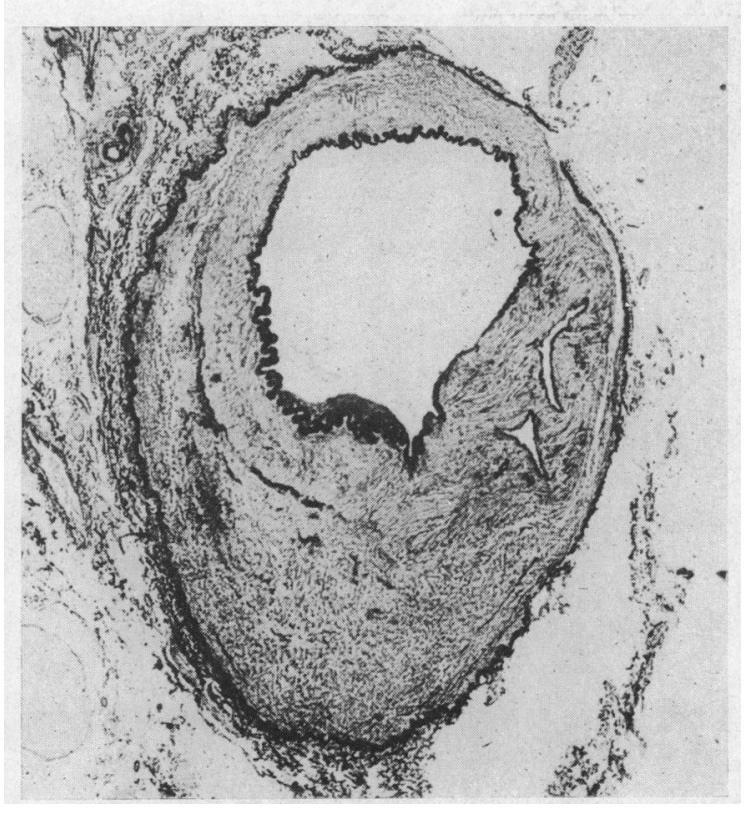

Fig. 2.-Case 1. Main renal artery showing marked subadventitial fibrosis (below) with external elastica fibres on the inner as well as the outer aspect of the fibrous zone. Several sinusoidal vessels are present in the media (right). (Hart-van Gieson. $\times 21$.)

occurring entirely within the external elastic and to be associated with fragmentation of its fibrils. The internal elastica was also disrupted at several points. The main branches of the renal artery and the aberrent artery showed no lesion.

Case 2. A 19-year-old journalist was admitted to hospital in November 1960 because of pain in the left hypochondrium. It was thought that this might have been due to a urinary tract infection but urinalysis was normal. A maternal aunt had hypertension. There was no previous history of renal disease. His blood pressure was $170 / 115 \mathrm{~mm}$. Hg. Routine examination was not otherwise remarkable. An intravenous pyelogram showed the right kidney to be $1.9 \mathrm{~cm}$. smaller than the left in bipolar diameter. The calyceal pattern was normal but the concentration of contrast was greater on the right side. In 1962 he developed morning headache and exertional dyspnoea, and when he presented to this hospital in July 1963 the blood pressure was 180/120 $\mathrm{mm}$. Hg. Venous pressure was normal, the heart was not enlarged but the heart sounds were accentuated. The retinal appearances were normal. The femoral pulses were not delayed. The kidneys were not palpable, but a continuous high-pitched right subcostal bruit was heard. A chest radiograph showed a normal cardiac outline but the electrocardiogram indicated left ventricular hypertrophy. Catecholamine excretion was $4.3 \mu \mathrm{g}$. $/ 24 \mathrm{hr}$. Blood urea was $21 \mathrm{mg}$. $/ 100 \mathrm{ml}$., serum potassium $3.7 \mathrm{mEq} /$ litre and $\mathrm{CO}_{2} \mathrm{CP} 24 \mathrm{mEq} / \mathrm{litre}$.

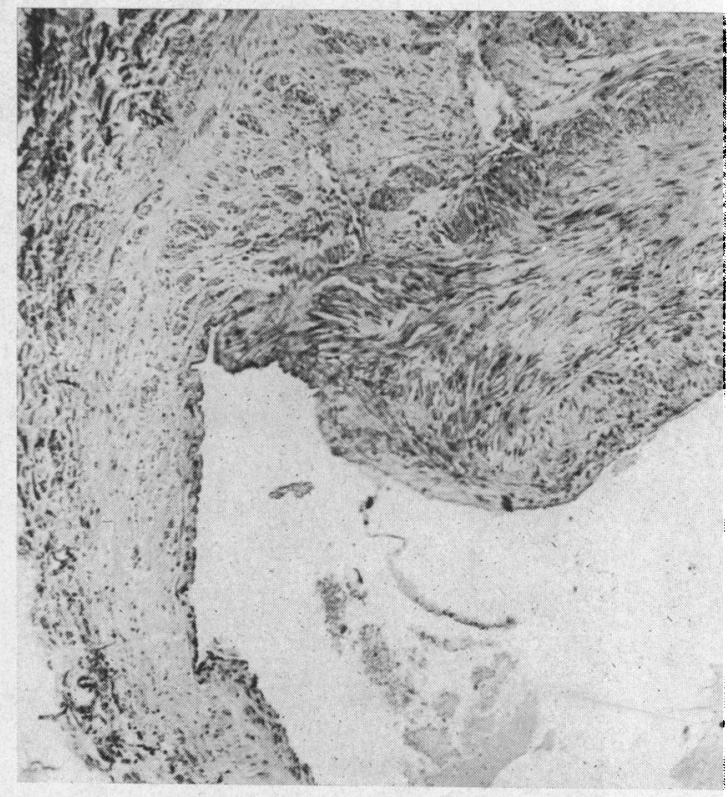

FIG. 3.-Case 1. Main renal artery, showing thickened zone with hyperplastic media (above) and gross thinning and fibrosis of the wall (below). Occasional bundles of medial muscle are seen in the fibrotic areas. (H. and E. $\times 44$.)

Urinalysis was normal. An intravenous pyelogram was similar to that obtained in 1960. A radio-hippuran renogram showed peak counts over the right renal area to be 26 per cent less than those over the left renal area, indicating asymmetry of renal function. Differential renal function studies during water diuresis showed a 92 per cent greater tubular reabsorption of sodium by the right kidney than by the left, suggesting right renal artery stenosis. A renal arteriogram (Fig. 4) showed a $1.5 \mathrm{~cm}$. stenosed segment of the main renal artery $2 \mathrm{~cm}$. from its origin. A collateral circulation of capsular vessels was demonstrated around the hilum of the kidney involving also the peri-ureteric vessel.

At operation in September 1963 the main right renal artery was narrowed to about half its original diameter at a point about $1 \mathrm{~cm}$. from its origin. The narrowed segment extended for a distance of $4 \mathrm{~cm}$. and was pulseless. A mean pressure gradient of $100 \mathrm{~mm}$. $\mathrm{Hg}$ was demonstrated across the stenosis. A venous graft reconstruction was performed. Following this procedure, however, a mean pressure gradient of $100 \mathrm{~mm}$. $\mathrm{Hg}$ was still demonstrable between the aorta and a primary branch artery. A right nephrectomy was then carried out. Convalescence was uneventful. The day following operation the blood pressure had fallen from 180/120 to $150 / 90 \mathrm{~mm}$. $\mathrm{Hg}$ and 18 months later the blood pressure was $130 / 90 \mathrm{~mm}$. Hg.

Pathology. The kidney weighed $137 \mathrm{~g}$. and showed no evidence of scarring or contraction. The wall of the main renal artery at the site of the graft was considerably 


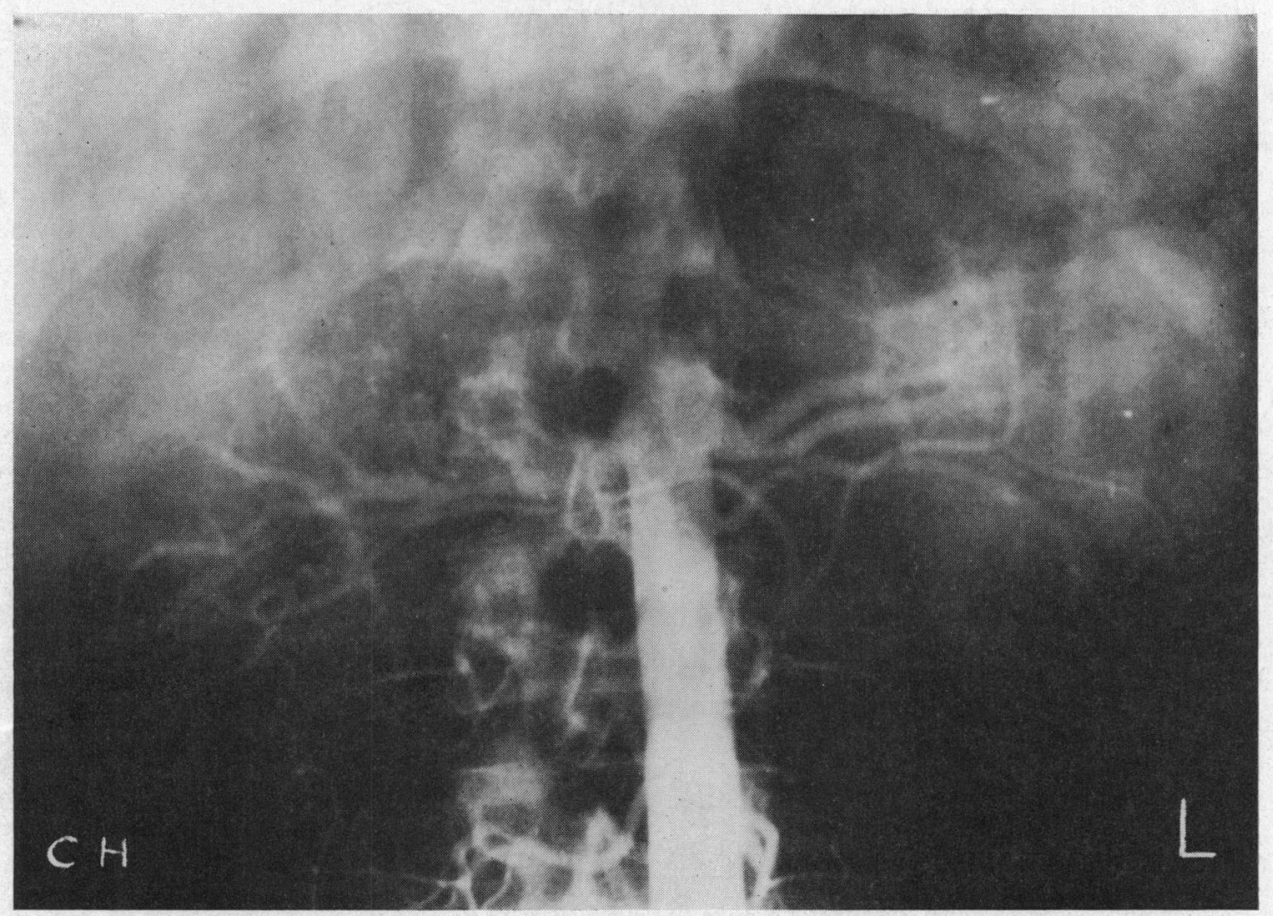

Fig. 4. - Renal arteriogram Case 2 showing a stenosed segment on distal third of the main right renal artery. A collateral circulation is present at the hilum.

thickened and very firm in consistency. The lumen was about half normal width. Distal to the graft and just before the bifurcation the artery lumen was of normal diameter and its main branches appeared normal.

Histology. The renal cortex showed tiny areas of subcapsular ischæmic scarring. The lobar arteries appeared normal but there was mild focal sclerosis of some smaller arteries and the arterioles. The glomeruli and tubules appeared unremarkable and the juxta-glomerular apparatuses were inconspicuous. The main renal artery in the region of the graft showed a thick clearly defined zone of dense fibrous tissue in the outer wall (Fig. 5), and there were moderate numbers of shrunken nuclei in the fibrous zone. Elastic tissue staining revealed numerous fibres of the external elastica on its outer aspect. Similar fibres, fewer in number and often showing considerable fragmentation and distortion, were present in some areas on the inner aspect and also in the substance of the fibrous zone. In a few areas fibrosis extended into the media in an irregular manner, and where the fibrous zone was thick the media was considerably narrowed. The media was otherwise well preserved and showed no hyperplasia. The internal elastic lamina was thickened in areas but not fragmented. There was no intimal fibrosis. The adventitia was normal in all areas. Distal to the stenosed segment the zone of subadventitial fibrosis became thin and could be followed for a few millimetres into the main branches of the renal artery before fading into the external elastica.

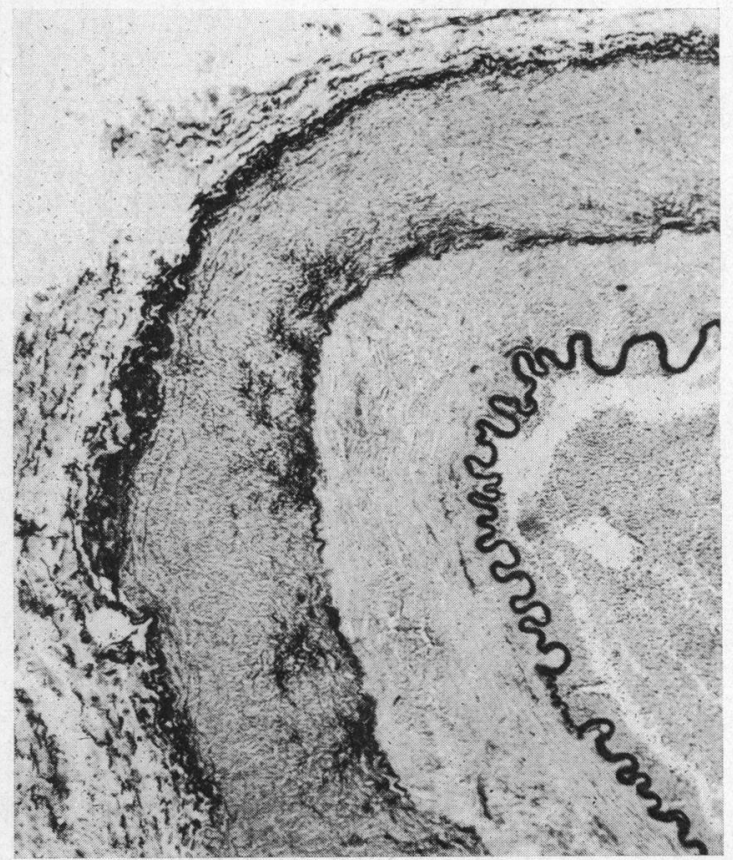

FIG. 5.-Case 2. Subadventitial fibrosis in the external elastica of main renal artery. (Hart-van Gieson. $\times 44$.) 


\section{Discussion}

The salient characteristic of the renal artery lesion in both cases was a conspicuous zone of fibrosis in the subadventitial area of the artery wall. In each the presence of fibres of the external elastica on both the inner and outer aspects of the fibrous zone as well as in its substance left little doubt that the fibrosis had developed primarily within the external elastic lamina. A similar relationship of fibrosis to this lamina was noted in the 3 cases of perimuscular fibrosis described by Wood and Borges (1963).

The occurrence of fibrosis restricted to this zone is difficult to explain. The sharply-defined character of the fibrosis in many areas and the wellpreserved state of the adjacent adventitia and especially of the media appear to exclude an inflammatory origin. A primary degeneration of elastic tissue is also unlikely as there seems no reason why such a degeneration should selectively attack the external elastica. In addition, both cases showed normal external elastic fibres on the outer aspect of the fibrosed zone. A third possibility is a dysplasia of fibrous tissue elements within the lamina. In this context it may be relevant that renal arteries of infants occasionally show a thin layer of myxomatous fibrous tissue which appears to occupy the same area of the vessel wall as subadventitial fibrosis (Wellington, 1963).

Several theories have been advanced concerning the aetiology of fibromuscular hyperplasia of the renal artery. These include a congenital origin (DeCamp and Birchall, 1958), an intrinsic basic defect of elastic tissue (Yendt et al., 1960), abnormal stretching of the renal arteries, especially in pregnancy (Yendt et al., 1960), and endocrine factors (Wylie et al., 1962). The evidence seems to favour a developmental origin for both fibromuscular hyperplasia and subadventitial fibrosis. Supporting this concept is the youth of many affected patients, the localization of the lesions to the distal two-thirds of the artery and its branches, the lack of resemblance to any known acquired arterial abnormality, and the occasional coexistence of the changes of subadventitial fibrosis and fibromuscular hyperplasia as in Case 1 . Evidence has recently appeared that fibromuscular hyperplasia may affect the extrarenal arteries (Palubinskas and Ripley, 1964).

In both of these young hypertensive patients the only clinical feature that distinguished them from the general hypertensive population was a subcostal bruit. This finding led to delineation of the stenotic lesion by renal arteriography. The frequency of such a bruit has been emphasized by Hunt et al. (1962) and Wylie et al. (1962), who also drew attention to its particular high-pitched quality, subcostal situation, and intermittency in the cases of fibrous and fibromuscular stenosis.

Disparity in renal size and function in the absence of the calyceal abnormalities of chronic pyelonephritis has been advocated as a screening test in the diagnosis of renal artery stenosis (Brown et al., 1960), but it is frequently unhelpful (Squire and Schlegel, 1959; Sutton et al., 1963). In the present cases the pyelogram appearances were not initially regarded as significant. The pathophysiology of renal artery stenosis, providing the theoretical basis for the functional changes seen in the pyelogram, has been considered by Brown et al. (1960) and Stamey et al. (1961). A more accurate evaluation of these changes, which demonstrate that an anatomical lesion is of functional significance in causing hypertension, may be obtained from differential renal function tests. The characteristic pattern of increased tubular reabsorption of sodium by the affected kidney was shown by both cases.

Visualization of the arterial lesion, however, depends on renal arteriography. In both cases involvement of the distal part of the main renal artery was shown and also the tortuous collateral vessels around the hilum, which are commonly present in fibrous and fibromuscular stenosis of the renal arteries. In Case 1 where subadventitial fibrosis and fibromuscular medial hyperplasia coexisted, the pathognomonic beaded appearance of the latter condition was well demonstrated.

The subadventitial fibrosis in Case 2 produced a tubular constriction of the vessel, and though not visible arteriographically or on gross inspection of the artery the lesion was seen histologically to extend into the primary branches.

Reconstructive arterial surgery rather than nephrectomy may be desirable in these non-atheromatous stenosing lesions of the renal arteries, because of our lack of knowledge of the natural history of the disease processes and the possibility of involvement of the renal artery supplying the opposite kidney, despite normal arteriographic appearances. However, the distal involvement of the main renal artery and the frequent extension into branch arteries often precludes reconstructive surgery (Spencer $e t$ al., 1961). Successful reconstructive procedures could not be carried out in either of these patients, but nephrectomy has resulted in a return of blood pressure to normal levels in both cases.

\section{SUMMARY}

The clinical, radiological, and pathological findings in 2 cases of hypertension due to renal artery stenosis are described. In both cases stenosis was 
due to subadventitial fibrosis. One showed, in addition, evidence of fibromuscular hyperplasia of the stenosed artery. Following nephrectomy the blood pressure fell in both cases and has remained at normal levels.

We are grateful to Dr. J. F. Pantridge for permission to publish the clinical details of these cases, and for his encouragement and advice.

\section{REFERENCES}

Brown, J. J., Owen, K., Peart, W. S., Robertson, J. I. S., and Sutton, D. (1960). The diagnosis and treatment of renal-artery stenosis. Brit. med. f., 2, 327.

D'Abreu, F., and Strickland, B. (1962). Developmental renal artery stenosis. Lancet, $2,517$.

DeCamp, P. T., and Birchall, R. (1958). Recognition and treatment of renal arterial stenosis associated with hypertension. Surgery, 43, 134.

Hunt, J. C., Harrison, E. G., Jr., Kincaid, O. W., Bernatz, P. E., and Davis, G. D. (1962). Idiopathic fibrous and fibromuscular stenosis of the renal arteries associated with hypertension. Proc. Mayo Clin., 37, 181.

McCormack, L. J. (1961). Vascular changes in hypertension. Med. Clin. N. Amer., 45, 247.

Morris, G. C., Jr., and De Bakey, M. E. (1962). Diagnosis of renal vascular disease. Amer. F. Cardiol., 9, 756.
Palubinskas, A. J., and Ripley, H. R. (1964). Fibromuscular hyperplasia in extrarenal arteries. Radiology, 82, 451.

Spencer, F. C., Stamey, T. A., Bahnson, H. T., and Cohen, A. (1961). Diagnosis and treatment of hypertension due to occlusive disease of the renal artery. Ann. Surg., 154, 674.

Squire, L. F., and Schlegel, J. U. (1959). Pyelography in renal disease with hypertension. Correlation between pyelographic findings and differential renal function studies. Radiology, 73, 849.

Stamey, T. A., Nudelman, I. J., Good, P. H., Schwentker, F. N., and Hendricks, F. (1961). Functional characteristics of renovascular hypertension. Medicine (Baltimore), 40, 347.

Sutton, D., Brunton, F. J., Foot, E. C., and Guthrie, J. (1963). Fibromuscular, fibrous and non-atheromatous renal artery stenosis and hypertension. Clin. Radiol, 14, 381.

Wellington, J. S. (1963). Fibromuscular hyperplasia of renal arteries in hypertension. Amer. F. Path., 43, 955.

Wood, C., and Borges, F. J. (1963). Perimuscular fibrosis of renal arteries with hypertension. Arch. intern. Med., 112, 79.

Wylie, E. J., Perloff, D., and Wellington, J. S. (1962). Fibromuscular hyperplasia of the renal arteries. Ann. Surg., 156, 592.

Yendt, E. R., Kerr, W. K., Wilson, D. R., and Jaworski, Z. F. (1960). The diagnosis and treatment of renal hypertension with special reference to a case of hypertension due to stenosis of both renal arteries. Amer. F. Med. 28, 169. 